\title{
Impact of cannabis use on thalamic volume in people at familial high risk of schizophrenia
}

Killian A. Welch, Andrew C. Stanfield, Andrew M. McIntosh, Heather C. Whalley, Dominic E. Job, Thomas W. Moorhead, David G. C. Owens, Stephen M. Lawrie and Eve C. Johnstone

\section{Background}

No longitudinal study has yet examined the association between substance use and brain volume changes in a population at high risk of schizophrenia.
Aims
To examine the effects of cannabis on longitudinal thalamus and amygdala-hippocampal complex volumes within a population at high risk of schizophrenia.

\section{Method}
Magnetic resonance imaging scans were obtained from individuals at high genetic risk of schizophrenia at the point of entry to the Edinburgh High-Risk Study (EHRS) and approximately 2 years later. Differential thalamic and amygdala-hippocampal complex volume change in high-risk individuals exposed $(n=25)$ and not exposed $(n=32)$ to cannabis in the intervening period was investigated using repeated-measures analysis of variance.

\section{Results}

Cannabis exposure was associated with bilateral thalamic volume loss. This effect was significant on the left $(F=4.47$, $P=0.04)$ and highly significant on the right $(F=7.66$, $P=0.008)$. These results remained significant when individuals using other illicit drugs were removed from the analysis.

\section{Conclusions}

These are the first longitudinal data to demonstrate an association between thalamic volume loss and exposure to cannabis in currently unaffected people at familial high risk of developing schizophrenia. This observation may be important in understanding the link between cannabis exposure and the subsequent development of schizophrenia.

\section{Declaration of interest}

None.
Studies of brain morphology in schizophrenia have provided overwhelming evidence of abnormal structural anatomy in people with the disorder. Two of the most consistently demonstrated structural abnormalities in schizophrenia are thalamic and hippocampal volume reduction. ${ }^{1,2}$ The point in illness development at which these abnormalities arise, and their relationship to established risk factors, has however been more difficult to ascertain.

Cannabis is an environmental factor for which there is considerable evidence of risk-modifying effects. ${ }^{3}$ Although there have been no consistent reports of brain structural abnormalities in association with use of this drug by the healthy population, ${ }^{4}$ studies have reported grey matter loss in association with cannabis use in people with established schizophrenia (e.g. Rais et $a l^{5}$ ). Furthermore, an earlier analysis of baseline data from the Edinburgh High-Risk Study (EHRS) found that people who are well but at genetically high risk of schizophrenia also exhibited structural brain abnormalities in association with cannabis use. ${ }^{6}$ In the current study we sought to examine the effects of cannabis on longitudinal thalamic and amygdala-hippocampal complex volumes within this high-risk population.

\section{Method}

\section{Participants}

Data were collected on people at elevated risk of schizophrenia as part of the EHRS. Details of this recruitment process have been described previously. ${ }^{7}$ In brief, individuals with schizophrenia, with a family history of schizophrenia and with relatives in adolescence/early adulthood, were identified from hospital case records. We then approached relatives aged 16-25 who were at high risk of schizophrenia: those who agreed to participate were given a detailed clinical, neuropsychological and brain imaging assessment. Assessments were repeated after approximately 2 years in consenting participants who had enrolled in the first 2 years of the study. As part of this repeat assessment, use of alcohol, tobacco and illicit drugs (including cannabis) in the interim period was ascertained by self-report. Exposures in this period were dichotomised as follows: cannabis use during this period or not; alcohol use exceeding UK government recommendations during this period (greater than 14 units/week for women and 21 units/week for men) or not; ecstasy use during this period or not; amphetamine use during this period or not; tobacco smoker during this period or not. The choice of a dichotomous rather than continuous measure of drug and alcohol use reflected the manner in which drug/alcohol use was recorded at the data collection stage.

\section{Rationale for focusing on the thalami and amygdala-hippocampal complex}

Previous reports from the EHRS have indicated that people who are clinically well but at genetically high risk of schizophrenia have reduced thalamic volume compared with controls, ${ }^{8}$ and studies have established thalamic reduction as a measure of genetic liability to psychosis. ${ }^{9,10}$ Further thalamic reductions occur between the vulnerability state and frank psychosis. ${ }^{11}$

In keeping with the possibility that cannabis may contribute to these changes, there are data suggesting that the thalamus is directly influenced by exposure to the drug. Cannabinoid 1 (CB1) receptors are expressed in this brain region, with highest levels of binding in the mediodorsal and anterior complex nuclei, ${ }^{12}$ regions which connect to cortical association areas consistently implicated in schizophrenia. ${ }^{13}$ Changes in thalamic regional cerebral blood flow secondary to cannabis consumption have also been reported, ${ }^{14,15}$ and animal studies find that cannabis consumption has robust and reproducible effects on the function 
of several thalamic subregions. ${ }^{16}$ We believe that the data outlined above provide a strong rationale for examining the effect of cannabis consumption on thalamic volume in this population, and this is the primary focus of this study. It is the case, however, that the amygdala-hippocampal complex has also been shown to undergo volume loss during transition from at-risk state to schizophrenia, ${ }^{17}$ and expresses a high density of CB1 receptors. ${ }^{18}$ For these reasons, as well as to ascertain whether any thalamic volume loss observed in the thalami was specific to this structure, we also examined the effect of cannabis consumption on volume of the amygdala-hippocampal complex. We have not extended the analysis to other structures, as this would have led to issues of multiple comparisons.

\section{Magnetic resonance imaging and analysis}

Each participant underwent magnetic resonance imaging (MRI) scanning on a $1 \mathrm{~T}$ Siemens (Erlangen, Germany) Magnetom scanner both at baseline $\left(T_{1}\right)$ and follow-up $\left(T_{2}\right)$. Details of image acquisition and processing have been given elsewhere. ${ }^{19}$ Image processing used the software package Analyze version 7.5 for UNIX (Mayo Foundation, Rochester, Minnesota, USA) to outline the thalamus and ascertain its volume. ${ }^{19}$

The thalami were outlined by naturalistic boundaries, as described in previous studies. ${ }^{19,20}$ All thalamic nuclei were grouped together, as MRI resolution makes distinction between the individual thalamic nuclei difficult. ${ }^{19}$ Additionally, given the technical difficulties inherent in separating the amygdala from the hippocampus at $1 \mathrm{~T}$, the amygdala-hippocampal complex was also traced as a unitary structure. Volumetric image processing was done by three investigators. The intraclass correlation coefficient between raters for the thalami was 0.84 . For the amygdala-hippocampal complex it was 0.82 .

\section{Statistical analysis}

Demographics and clinical variables were compared between high-risk subgroups that did and did not consume cannabis in the interim period, with the independent $t$-test and the $\chi^{2}$-test. Additionally, baseline whole-brain and region of interest volumes were compared between these two subgroups using the independent $t$-test.

Any differential thalamic or amygdala-hippocampal complex volume change in high-risk individuals exposed and not exposed to cannabis in the interim period was examined for right and left structures separately, using repeated-measures analysis of variance and looking for cannabis exposure $\times$ time interactions. Structure volume at $T_{1}$ and $T_{2}$ were entered as the dependent variable, gender and exposure status to cannabis, alcohol, tobacco, ecstasy and amphetamines were included as fixed factors, and age at first assessment and time span between assessments at $T_{1}$ and $T_{2}$ were entered as covariates. Standardised residuals were checked for normality. To facilitate interpretation of the findings, mean rate of change in structure volume was also calculated from the raw data. A small number of participants had used illicit drugs other than those listed above. Repeated-measures analysis was therefore re-run excluding any individuals with a history of use of any substance other than alcohol, cannabis, tobacco, ecstasy and amphetamines. None of the participants who did not consume cannabis between the two time points had used either ecstasy or amphetamines, whereas substantial numbers of individuals using cannabis had used one of these drugs. Given this imbalance of variables, and the evidence that ecstasy in particular may be specifically thalamotoxic, ${ }^{21}$ repeated-measures analysis was also run excluding any participants who had used either of these two drugs. All analyses were conducted in SPSS (version 14) for Windows.

It was conceivable that change occurring in structure volume in association with cannabis consumption was simply part of a more generalised effect. To ascertain whether any volume changes were specific to the structures investigated, repeated-measures analysis was re-run with addition of rate of change of whole-brain volume ([WBV2 - WBV1]/time between assessments) included as an additional covariate.

\section{Results}

Scans were obtained at both time points in 66 individuals at familial high risk of schizophrenia. Substance misuse data were available for 57 of these individuals of whom 25 consumed cannabis between the two assessments.

Demographic and clinical characteristics of these individuals who did and did not consume cannabis in the interim period are shown in Table 1 . In addition to the substances detailed in Table 1, two participants used opiates, two cocaine and three lysergic acid diethylamide (LSD) in the period between scans; in all cases these people were in the cannabis exposure group. At baseline there was no significant difference in whole-brain, thalamic or amygdala-hippocampal complex volumes when comparing individuals who did or did not use cannabis in the interim period. Additionally, the two groups were reasonably balanced at baseline in terms of age, gender and scores on the Rust Inventory of Schizotypal Cognitions, a measure of mild positive psychotic symptoms. ${ }^{22}$

The mean raw absolute volume change in the structures of interest together with mean rate of volume change ([structure volume at $T_{2}$-structure volume at $\left.T_{1}\right] /$ time between scans) in both the cannabis exposed and non-exposed groups is shown in Table 2. Cannabis exposure $\times$ time interactions are also shown, after inclusion of the covariates detailed in the accompanying text. As can be seen, cannabis exposure is associated with bilateral thalamic volume loss, this effect being significant on the left $(F=4.47, P=0.04)$ and highly significant on the right $(F=7.66$, $P=0.008)$. Analysis was repeated, excluding the seven participants who consumed illicit drugs other than cannabis, ecstasy or amphetamines in the period of interest; the cannabis exposure $x$ time interaction remained significant on both the right- and lefthand side. It was also re-run excluding the 14 people who had used either ecstasy or amphetamine in the period of interest; again, the cannabis exposure $\times$ time interaction remained significant bilaterally. Additionally, the primary analysis was also re-run with the inclusion of rate of change of whole-brain volume as an additional covariate. Once again, the cannabis exposure $\times$ time interaction remained significant on both sides. In contrast to the effects seen in the thalami, those in the amygdala-hippocampal complex were non-significant.

\section{Discussion}

The present study finds thalamic volume reduction over time in a population at high familial risk of schizophrenia who consume cannabis. This volume loss is not observed in individuals at high familial risk of schizophrenia who remain cannabis-free during the inter-scan interval.

Evidence for a role in the aetiology of schizophrenia is (arguably) stronger for cannabis than for any other putative environmental risk factor. It has also been demonstrated that the rate of grey matter loss in people recently diagnosed with schizophrenia is accelerated in those who consume the drug, ${ }^{5}$ an 


\begin{tabular}{|c|c|c|c|}
\hline & No cannabis use $(n=32)$ & Cannabis use $(n=25)$ & $P^{\mathrm{a}}$ \\
\hline Age at first assessment, years: mean (s.d.) & $21.11(2.87)$ & $21.76(2.52)$ & 0.38 \\
\hline Gender, male:female & $15: 17$ & $15: 10$ & $0.33^{b}$ \\
\hline Handedness, right:left:both & 28:4:0 & 21:2:2 & $0.24^{b}$ \\
\hline Exceed recommended maximum alcohol consumption, $n$ & 4 & 7 & $0.18^{c}$ \\
\hline Smoke tobacco, $n$ & 8 & 18 & $<0.001^{\mathrm{b}}$ \\
\hline Use ecstasy, $n$ & 0 & 9 & $<0.001^{c}$ \\
\hline Use amphetamines, $n$ & 0 & 10 & $<0.001^{c}$ \\
\hline Days between assessments, mean (s.d.) & $648.38(128.06)$ & $679.12(206.85)$ & 0.49 \\
\hline Rate of change in whole-brain volume, $\mathrm{mm}^{3} /$ day: mean (s.d.) & $-13.49(43.74)$ & $-0.27(68.20)$ & 0.40 \\
\hline Baseline whole-brain volume, $\mathrm{cm}^{3}$ : mean (s.d.) & $1349.13(127.07)$ & $1362.85(138.86)$ & 0.70 \\
\hline Baseline right thalamic volume, $\mathrm{cm}^{3}$ : mean (s.d.) & $6.01(0.77)$ & $6.23(0.87)$ & 0.29 \\
\hline Baseline left thalamic volume, $\mathrm{cm}^{3}$ : mean (s.d.) & $6.15(0.79)$ & $6.29(0.91)$ & 0.55 \\
\hline Baseline right AHC volume, $\mathrm{cm}^{3}$ : mean (s.d.) & $4.74(0.68)$ & $4.85(0.97)$ & 0.52 \\
\hline Baseline left AHC volume, $\mathrm{cm}^{3}$ : mean (s.d.) & $4.60(0.52)$ & $4.60(0.63)$ & 0.98 \\
\hline Rust Inventory of Schizotypal Cognitions baseline score, mean (s.d.) & $25.44(9.94)$ & $27.92(11.70)$ & 0.39 \\
\hline
\end{tabular}

observation in keeping with reports that cannabis consumption may affect the course of established schizophrenia. ${ }^{23,24}$ Ours are, however, the first longitudinal data to demonstrate that this association between structure volume loss and exposure to cannabis is present in people at genetically high risk of developing schizophrenia who are currently not psychotic. This observation may be important in understanding the link between cannabis exposure and the subsequent development of schizophrenia.

\section{Importance of the thalamus}

The structure in which we observe this progressive cannabisassociated volume loss, the thalamus, is believed to function as an information-processing and relay station. It is a conduit for bidirectional flow of signals between cortical and subcortical regions, links different cortical regions via transthalamic pathways and is a point of convergence for frontostriatal and cerebellothalamo-cortical circuits. ${ }^{25}$ Given this role of interconnecting diverse brain regions, it would be expected that thalamic lesions would have widespread consequences. It may also be expected, given that schizophrenia is increasingly regarded as a disorder of connectivity, ${ }^{26}$ that these may resemble schizophrenia. This is indeed what has been demonstrated by studies examining the consequences of thalamic vascular insults; such lesions resulting in a variety of schizophrenia-like presentations, with negative symptoms being particularly prominent. ${ }^{25}$

The prediction that thalamic dysfunction will result in impairment of proper communication between different brain regions is central to many of the models proposed to explain the pathophysiology of schizophrenia. Andreasen et al, for example, proposed that a disruption in prefrontal-thalamiccerebellar circuitry may contribute to the cognitive disturbances that are characteristic of the condition. ${ }^{27,28}$ In keeping with this, functional imaging has previously demonstrated that this network is indeed disrupted in the EHRS cohort, ${ }^{29}$ a population known to exhibit subtle impairments in the cognitive domains affected in schizophrenia. ${ }^{30,31}$ Other neural models have focused on the connections the thalamus has with the dorsolateral prefrontal cortex and the potential impact of thalamic dysfunction on working memory. ${ }^{32,33}$ Still others have emphasised the potential role of the thalamus in impaired sensory gating. ${ }^{34}$ Intriguingly, neuropsychological testing has demonstrated that within a population with schizophrenia, thalamic volume is positively associated with global neuropsychological performance as well as scores in the specific domains of motor, language and executive functioning. ${ }^{35}$

Although diverse, the models outlined have a common implication: given the extensive connections of the thalamus, even

Table 2 Comparison of structure volume changes between scans in individuals at high familial risk of schizophrenia, exposed and not exposed to cannabis

\begin{tabular}{|c|c|c|c|c|c|}
\hline & \multicolumn{2}{|c|}{$\begin{array}{c}\text { Absolute change } \\
\text { (scan } 2-\text { scan 1), } \mathrm{mm}^{3} \text { : mean (s.d.) }\end{array}$} & \multicolumn{2}{|c|}{$\begin{array}{c}\text { Rate of change } \\
\text { ([scan 2-scan 1]/time), } \mathrm{mm}^{3} / \text { day: mean (s.d.) }\end{array}$} & \multirow{2}{*}{$\begin{array}{l}\text { Exposure } \times \text { time } \\
\text { interaction }^{\mathrm{a}}\end{array}$} \\
\hline & No cannabis exposure & Cannabis exposure & No cannabis exposure & Cannabis exposure & \\
\hline Right thalamus & $32.84(509.35)$ & $-264.48(621.70)$ & $0.06(0.80)$ & $-0.36(0.92)$ & $F=7.66, P<0.01$ \\
\hline Left thalamus & $26.66(667.58)$ & $-181.39(621.70)$ & $0.07(1.00)$ & $-0.22(1.01)$ & $F=4.47, P=0.04$ \\
\hline Right amygdala-hippocampal complex & $147.77(563.94)$ & $175.31(432.19)$ & $0.18(0.95)$ & $0.19(0.66)$ & $F=0.71, P=0.40$ \\
\hline Left amygdala-hippocampal complex & $-121.37(488.70)$ & $-4.43(384.77)$ & $-0.23(0.81)$ & $-0.03(0.59)$ & $F=0.01, P=0.92$ \\
\hline
\end{tabular}


relatively minor abnormalities of this structure could potentially have dramatic consequences. Even though a genetic propensity to schizophrenia is itself associated with reduced thalamic volume, further volume reductions occur between the vulnerability state and frank psychosis. ${ }^{11}$ By demonstrating that cannabis use by a vulnerable population is associated with thalamic volume loss, the current findings raise the possibility that this drug may be a factor which increases the likelihood of such abnormalities arising. This may lead to a worsening of previously subtle symptomatology and ultimately influence the risk of transition to schizophrenia.

\section{Self-medication}

Although substantial data suggest that cannabis use is a risk factor for schizophrenia, a frequently posited non-causative explanation for its association with the condition is the selfmedication hypothesis. In applying this explanation to the current data, it would be argued that the experience of schizophrenic-type symptomatology leads to both cannabis use and thalamic volume loss. We believe such an explanation unlikely, it being undermined by the fact that individuals in this study were all well between the two assessment points. Although some participants in the EHRS did show transient or partial psychotic symptoms, there was no significant difference in ratings on the Rust Inventory of Schizotypal Cognitions at baseline between those who did and did not use cannabis between the scan points. The possibility that biological processes associated with the development of psychotic symptoms is driving both the thalamic volume loss and cannabis use (and hence the association between the two) also seems unlikely given previous findings from the EHRS demonstrating no thalamic volume change when those with or without psychotic symptoms are compared. ${ }^{36}$ Consequently, we feel that the most likely explanation for the effects seen is that they are indeed secondary to cannabis exposure, the current data therefore strengthening the supposition that cannabis can play an aetiological role in the development of schizophrenia.

\section{Strengths and limitations}

A limitation of this study is that we did not examine the impact of cannabis use in a non-high-risk comparator group, raising the possibility that the effects observed are a normal consequence of cannabis use rather than specific to those at elevated genetic risk of schizophrenia. Unfortunately, too few of the control participants in the EHRS (only four) consumed cannabis in the interim period for such an analysis to be statistically meaningful. As discussed in the introduction, however, there is little evidence of cannabis-associated brain structural abnormalities in the healthy population. ${ }^{4}$ It therefore seems highly unlikely that the effects we observe would also be seen in a population not at genetically high risk of schizophrenia.

Further limitations of the study are that it was inadequately powered to establish whether thalamic volume loss in association with cannabis use predicts subsequent schizophrenia (although we do know that seven of the high-risk participants did develop the condition at some point after the second scan), or to explore dose-response effects.

We were also unable to establish whether volume loss was attributable to changes in specific thalamic nuclei. This was outwith the scope of this particular research, but clearly would be of significant interest. Not least, it would suggest the regions in which we may expect to find the cannabis-associated abnormalities of brain connectivity that may be central in understanding how thalamic abnormalities contribute to the symptomatology of schizophrenia. Previous research using a statistical modelling approach to analyse shape reported that inward deformations of the anterior and posterior thalamus are more pronounced in people with schizophrenia than their unaffected relatives (who were intermediate between those with schizophrenia and healthy controls). ${ }^{37}$ Additionally, post-mortem studies of people who had schizophrenia have observed reduced volume and/or neuronal numbers in the mediodorsal nucleus, anterior nucleus and pulvinar. ${ }^{38,39}$ These findings have generally been in agreement with structural MRI studies. ${ }^{40}$ Clearly, more accurate localisation of the regions of thalamic volume loss secondary to cannabis use, together with identification of any cannabis-associated thalamocortical connectivity changes, will be an important focus of future work in this field. As this paper demonstrates, negative findings when such studies are undertaken in the normal cannabis-using population does not preclude the possibility that significant effects are seen in populations with a genetic loading for schizophrenia.

\section{Killian A. Welch, MD, MRCPsych, Robert Ferguson Unit, Astley Ainslee Hospital, Edinburgh and Division of Psychiatry, University of Edinburgh, Royal Edinburgh Hospital; Andrew C. Stanfield, MPhil, MRCPsych, Andrew M. McIntosh, MD, MRCPsych, Heather C. Whalley, PhD, Dominic E. Job, PhD, Thomas w. Moorhead, PhD, David G. C. Owens, MD, FRCPsych, Stephen M. Lawrie, MD, FRCPsych, Eve C. Johnstone, MD, FRCPsych, Division of Psychiatry, School of Molecular and Clinical Medicine, University of Edinburgh, Royal Edinburgh Hospital, UK \\ Correspondence: Killian A. Welch, Robert Ferguson Unit, Astley Ainslee Hospital, Edinburgh and Division of Psychiatry, University of Edinburgh, Royal Edinburgh Hospital, Edinburgh EH10 5HF, UK. Email: kwelch1@staffmail.ed.ac.uk}

First received 29 Nov 2010, final revision 27 May 2011, accepted 16 Jun 2011

\section{Funding}

This work was funded by the UK Medical Research Council and the Dr Mortimer and Theresa Sackler Foundation.

\section{References}

1 Crespo-Facorro B, Roiz-Santiáñez R, Pelayo-Terán JM, Rodríguez-Sánchez JM, Pérez-Iglesias R, González-Blanch C, et al. Reduced thalamic volume in first-episode non-affective psychosis: correlations with clinical variables, symptomatology and cognitive functioning. Neuroimage 2007; 35: 1613-23.

2 Shenton ME, Dickey CC, Frumin M, McCarley RW. A review of MRI findings in schizophrenia. Schizophr Res 2001; 49: 1-52.

3 Arseneault L, Cannon M, Witton J, Murray RM. Causal association between cannabis and psychosis: examination of the evidence. Br J Psychiatry 2004; 184: $110-7$.

4 Martín-Santos R, Fagundo AB, Crippa JA, Atakan Z, Bhattacharyya S, Allen P, et al. Neuroimaging in cannabis use: a systematic review of the literature. Psychol Med 2010; 40: 383-98.

5 Rais M, Cahn W, Van Haren N, Schnack H, Caspers E, Hulshoff Pol H, et al. Excessive brain volume loss over time in cannabis-using first-episode schizophrenia patients. Am J Psychiatry 2008; 165: 490-6.

6 Welch KA, McIntosh AM, Job DE, Whalley HC, Moorhead TW, Hall J, et al. The impact of substance use on brain structure in people at high risk of developing schizophrenia. Schizophr Bull 2010; Mar 11. Epub ahead of print.

7 Hodges A, Byrne M, Grant E, Johnstone E. People at risk of schizophrenia. Sample characteristics of the first 100 cases in the Edinburgh High-Risk Study. Br J Psychiatry 1999; 174: 547-53.

8 Lawrie SM, Whalley HC, Abukmeil SS, Kestelman JN, Donnelly L, Miller P, et al. Brain structure, genetic liability, and psychotic symptoms in subjects at high risk of developing schizophrenia. Biol Psychiatry 2001; 49: 811-23.

9 McDonald C, Bullmore E, Sham P, Chitnis X, Suckling J, MacCabe J, et al. Regional volume deviations of brain structure in schizophrenia and psychotic bipolar disorder. Computational morphometry study. Br J Psychiatry 2005; 186: 369-77.

10 McIntosh AM, Job DE, Moorhead WJ, Harrison LK, Whalley HC, Johnstone EC, et al. Genetic liability to schizophrenia or bipolar disorder and its relationship to brain structure. Am J Med Genet B Neuropsychiatr Genet 2006; 141B: 76-83. 
11 Chan RCK, Di X, McAlonan GM, Gong Q. Brain anatomical abnormalities in high-risk individuals, first-episode, and chronic schizophrenia: an activation likelihood estimation meta-analysis of illness progression. Schizophr Bull 2011; 37: 177-88.

12 Svíženská I, Dubový P, Šulcová A. Cannabinoid receptors 1 and 2 (CB1 and $\mathrm{CB} 2$ ), their distribution, ligands and functional involvement in nervous system structures: a short review. Pharmacol Biochem Behav 2008; 90: 501-11.

13 Shenton ME, Dickey CC, Frumin M, McCarley RW. A review of MRI findings in schizophrenia. Schizophr Res 2001; 49: 1-52.

14 Mathew RJ, Wilson WH, Coleman RE, Turkington TG, DeGrado TR. Marijuana intoxication and brain activation in marijuana smokers. Life Sci 1997; 60 2075-89.

15 O'Leary DS, Block RI, Koeppel JA, Flaum M, Schultz SK, Andreasen NC et al. Effects of smoking marijuana on brain perfusion and cognition. Neuropsychopharmacology 2002; 26: 802-16.

16 Freedland CS, Whitlow CT, Miller MD, Porrino LJ. Dose-dependent effects of $\Delta$ 9-tetrahydrocannabinol on rates of local cerebral glucose utilization in rat. Synapse 2002; 45: 134-42.

17 Lawrie SM, McIntosh AM, Hall J, Owens DGC, Johnstone EC. Brain structure and function changes during the development of schizophrenia: the evidence from studies of subjects at increased genetic risk. Schizophr Bull 2008; 34 330-40.

18 Eggan SM, Lewis DA. Immunocytochemical distribution of the cannabinoid CB1 receptor in the primate neocortex: a regional and laminar analysis. Cereb Cortex 2007; 17: 175-91.

19 Whalley HC, Kestelman JN, Rimmington JE, Kelso A, Abukmeil SS, Best JJK, et al. Methodological issues in volumetric magnetic resonance imaging of the brain in the Edinburgh High Risk Project. Psychiatr Res 1999; 91: 31-44.

20 Lawrie SM, Whalley HC, Abukmeil SS, Kestelman JN, Donnelly L, Miller P, et al Brain structure, genetic liability, and psychotic symptoms in subjects at high risk of developing schizophrenia. Biol Psychiatry 2001; 49: 811-23.

21 de Win MML, Jager G, Booij J, Reneman L, Schilt T, Lavini C, et al. Neurotoxic effects of ecstasy on the thalamus. Br J Psychiatry 2008; 193: 289-96.

22 Rust J. The Rust Inventory of Schizotypal Cognitions (RISC). Schizophr Bull 1988; 14: 317-22.

23 Linszen DH, Dingemans PM, Lenior ME. Cannabis abuse and the course of recent-onset schizophrenic disorders. Arch Gen Psychiatry 1994; 51: 273-9.

24 Zammit S, Moore THM, Lingford-Hughes A, Barnes TRE, Jones PB, Burke M et al. Effects of cannabis use on outcomes of psychotic disorders: systematic review. Br J Psychiatry 2008; 193: 357-63.

25 Byne W, Hazlett EA, Buchsbaum MS, Kemether E. The thalamus and schizophrenia: current status of research. Acta Neuropathol 2009; 117 347-68
26 Friston KJ, Frith CD. Schizophrenia: a disconnection syndrome? Clin Neurosci 1995; 3: 89-97.

27 Andreasen NC, Paradiso S, O'Leary DS. 'Cognitive dysmetria' as an integrative theory of schizophrenia: a dysfunction in cortical-subcorticalcerebellar circuitry? Schizophr Bull 1998; 24: 203-18.

28 Andreasen NC, Nopoulos P, O'Leary DS, Miller DD, Wassink T, Flaum M. Defining the phenotype of schizophrenia: cognitive dysmetria and its neural mechanisms. Biol Psychiatry 1999; 46: 908-20.

29 Whalley HC, Simonotto E, Flett S, Marshall I, Ebmeier KP, Owens DGC, et al. fMRI correlates of state and trait effects in subjects at genetically enhanced risk of schizophrenia. Brain 2004; 127: 478-90.

30 Byrne M, Hodges A, Grant E, Owens DC, Johnstone EC. Neuropsychological assessment of young people at high genetic risk for developing schizophrenia compared with controls: preliminary findings of the Edinburgh High Risk Study (EHRS). Psychol Med 1999; 29: 1161-73.

31 O'Connor M, Harris JM, McIntosh AM, Owens DGC, Lawrie SM, Johnstone EC. Specific cognitive deficits in a group at genetic high risk of schizophrenia. Psychol Med 2009; 39: 1649-55.

32 Carlsson M, Carlsson A. Schizophrenia: a subcortical neurotransmitter imbalance syndrome? Schizophr Bull 1990; 16: 425-32.

33 Jones EG. Cortical development and thalamic pathology in schizophrenia. Schizophr Bull 1997; 23: 483-501.

34 Mega MS, Cummings JL. Frontal-subcortical circuits and neuropsychiatric disorders. J Neuropsychiatry Clin Neurosci 1994; 6: 358-70.

35 Coscia DM, Narr KL, Robinson DG, Hamilton LS, Sevy S, Burdick KE, et al. Volumetric and shape analysis of the thalamus in first-episode schizophrenia. Hum Brain Mapp 2009; 30: 1236-45.

36 Lawrie SM, Whalley HC, Abukmeil SS, Kestelman JN, Miller P, Best JJK, et al. Temporal lobe volume changes in people at high risk of schizophrenia with psychotic symptoms. Br J Psychiatry 2002; 181: 138-43.

37 Harms MP, Wang L, Mamah D, Barch DM, Thompson PA, Csernansky JG Thalamic shape abnormalities in individuals with schizophrenia and their nonpsychotic siblings. J Neurosci 2007; 27: 13835-42.

38 Cronenwett WJ, Csernansky J. Thalamic pathology in schizophrenia. In Behavioral Neurobiology of Schizophrenia and Its Treatment (eds DA Kendall, S Alexander): 509-28. Springer-Verlag, 2010.

39 Young KA, Manaye KF, Liang CL, Hicks PB, German DC. Reduced number of mediodorsal and anterior thalamic neurons in schizophrenia. Biol Psychiatry 2000; 47: 944-53.

40 Sim K, Cullen T, Ongur D, Heckers S. Testing models of thalamic dysfunction in schizophrenia using neuroimaging. J Neural Transm 2006; 113: 907-28. 